\title{
EUROfusion
}

EUROFUSION WPJET1-PR(15)19

J-B. Girardo et al.

\section{Stabilization of Sawteeth with 3rd Harmonic Deuterium ICRF-Accelerated Beam in JET Plasmas}


"This document is intended for publication in the open literature. It is made available on the clear understanding that it may not be further circulated and extracts or references may not be published prior to publication of the original when applicable, or without the consent of the Publications Officer, EUROfusion Programme Management Unit, Culham Science Centre, Abingdon, Oxon, OX14 3DB, UK or e-mail Publications.Officer@euro-fusion.org".

"Enquiries about Copyright and reproduction should be addressed to the Publications Officer, EUROfusion Programme Management Unit, Culham Science Centre, Abingdon, Oxon, OX14 3DB, UK or e-mail Publications.Officer@euro-fusion.org".

The contents of this preprint and all other EUROfusion Preprints, Reports and Conference Papers are available to view online free at http://www.euro-fusionscipub.org. This site has full search facilities and e-mail alert options. In the JET specific papers the diagrams contained within the PDFs on this site are hyperlinked. 


\title{
Stabilization of Sawteeth with 3rd Harmonic Deuterium ICRF-Accelerated Beam in JET Plasmas
}

\author{
Jean-Baptiste Girardo, ${ }^{1,2}$ Sergei Sharapov, ${ }^{3}$ Rémi Dumont, ${ }^{2}$ Jacob Eriksson, ${ }^{4}$ Michael Fitzgerald, ${ }^{3}$ Xavier \\ Garbet, ${ }^{2}$ Nick Hawkes, ${ }^{3}$ Vasily Kiptily, ${ }^{3}$ Mervi Mantsinen, ${ }^{5,} 6$ Yanick Sarazin, ${ }^{2}$ Mireille Schneider, ${ }^{2}$ and JET \\ Contributors $^{\text {a) }}$ \\ 1) EUROfusion Consortium, JET, Culham Science Centre, Abingdon, OX14 3DB, \\ $U K$. \\ ${ }^{2)}$ CEA, IRFM, F-13108 Saint-Paul-lez-Durance, France. \\ 3) CCFE, Culham Science Centre, Abingdon, OX14 3DB, UK. \\ 4) Department of Physics and Astronomy, Uppsala University, Uppsala, Sweden. \\ 5) Catalan Institution for Research and Advanced Studies, Barcelona, Spain \\ ${ }^{6)}$ Barcelona Supercomputing Center, Barcelona, Spain
}

(Dated: April 30, 2015)

Results on sawtooth stabilization by fast particles in JET are presented. Those fast particles are produced by Neutral Beam Injection (NBI) and 3rd harmonic deuterium Ion Cyclotron Radio-Frequency (ICRF) heating, with a cut off energy around $2 \mathrm{MeV}$. Four sawteeth from four different discharges are compared. One of them is a monster sawtooth of $2.5 \mathrm{~s}$, during which the fast particle population and the $q$-profile are remarkably stable. In two discharges, the crash is triggered by tornado modes which expel the fast particles from within the $q=1$ surface, over time scales comparable to the sawtooth period. In the other two discharges (including the discharge exhibiting the monster sawtooth), no tornado modes occur; the crash is triggered by a fast (a few $10 \mathrm{~ms}$ ) occurring event, according to Porcelli's model.

\section{INTRODUCTION}

Sawteeth are a widely seen phenomenon exhibited by fusion plasmas when the safety factor $q$ drops below 1. They have been extensively studied, in conjunction with internal kink modes, neoclassical tearing modes and magnetic reconnection issues. ${ }^{1-5}$ It has been shown that, under certain circumstances, sawteeth oscillations can be stabilized by a kinetic contribution from energetic ions. ${ }^{6-14}$ The problem of sawtooth stabilization by fast ions is of major importance in view of the next step burning plasma experiments; the question of the optimal sawteeth frequency ${ }^{15}$ in plasmas with a large population of fusion-produced alpha particles remains open.

The purpose of the present paper is to report on very strong effect of sawtooth stabilization by fast deuterium (D) beam ions accelerated to about $100 \mathrm{keV}$ by Neutral Beam Injection (NBI), and then to the MeV energy range by 3rd harmonic Ion Cyclotron Resonance Heating (ICRH). Four representative sawteeth from four different JET discharges are studied. One of them, in discharge \#86775, is a $2.5 \mathrm{~s}$ long monster sawtooth, during which the fast particle population and the $q$-profile are remarkably stable. According to Porcelli's model, the event causing the sawtooth crash occurred on a short time scale (a few $10 \mathrm{~ms}$ ). The stabilization of a sawtooth by 3rd harmonic deuterium ICRH in JET was already studied in Ref. 16, in discharge \#74951. However, in that discharge, the fast particles did not exhibit a stable profile and the event causing the sawtooth crash

a) See the Appendix of F. Romanelli et al., Proceedings of the 25th IAEA Fusion Energy Conference 2014, Saint Petersburg, Russia occurred on a longer time scale (a few $100 \mathrm{~ms}$ ).

In the present study, the efficiency of sawtooth stabilization by the fast particles obtained in our experiment is assessed within Porcelli's model. We find that the stabilization provided by fast particles is strong, in accordance with the experiment. In particular, the model predicts higher stabilization in the case of discharge \#86775, which is the discharge where the monster sawtooth was observed. We then aim at understanding the reasons which cause the sawtooth crashes, while fast particles provide such a strong stabilization.

\section{PRESENTATION OF THE FOUR CONSIDERED JET DISCHARGES - FRAMEWORK OF THE PRESENT STUDY}

Four sawteeth, from four different discharges (\#86459, \#86762, \#86774 and \#86775), are considered in the present study. Those four discharges were performed in June and July 2014, on JET with Iter-Like Wall (ILW), ${ }^{17}$ at $B_{0} \sim 2.3 \mathrm{~T}, I \sim 2 \mathrm{MA}, R_{0} \sim 3 \mathrm{~m}$ and $a \sim 0.9 \mathrm{~m}$, where $B_{0}$ is the value of the magnetic field on the magnetic axis, $I$ is the value of the toroidal plasma current, $R_{0}$ is the major radius of the magnetic axis and $a$ is the minor radius of the plasma.

In all four cases, deuterium Neutral Beam Injection (NBI) and 3rd harmonic deuterium ICRH heating were performed. The plasma was deuterium only in one discharge, and deuterium - helium 3 in the three others. For each discharge, four ICRH antennas were used with frequencies between 51.4 and $51.8 \mathrm{MHz}$, bringing the NBIaccelerated particles from the $10-100 \mathrm{keV}$ range to the 

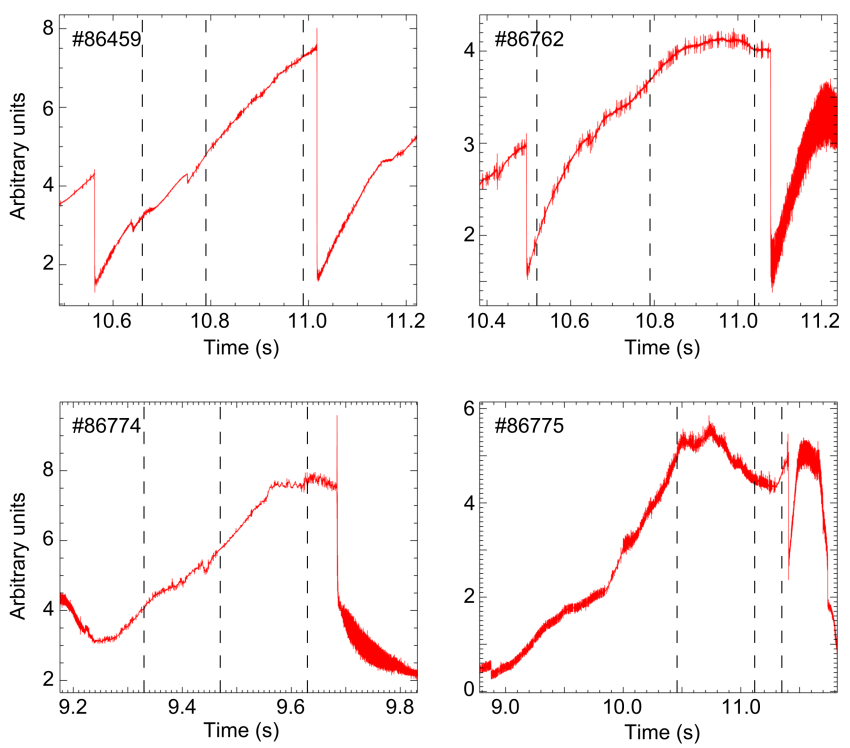

Figure 1. Soft X-rays signals for the four sawteeth studied in the present paper. Arbitrary units on y-axis. The time slices for which values are presented in table I are here marked with dashed lines.

\section{$\mathrm{MeV}$ range.}

On Figure 1, the soft X-ray signals corresponding to the four sawteeth are presented. The first sawtooth occurred in discharge \#86459, lasting about $0.4 \mathrm{~s}$. The second sawtooth was measured in discharge \#86762, with a duration of over $0.5 \mathrm{~s}$. The third sawtooth happened in discharge \#86774 and lasted about $0.4 \mathrm{~s}$. Finally, the fourth sawtooth occurred in discharge \#86775: it was a monster sawtooth, with a duration of over $2.4 \mathrm{~s}$, which was a record long sawtooth period for JET with ITER-like wall.

The present study is carried out in the framework of a theoretical model developed by Porcelli in Ref. 9. According to this model, a sawtooth remains stable as long as the potential energy functional $\delta \hat{W}=\delta \hat{W}_{\mathrm{MHD}}+\delta \hat{W}_{\mathrm{kin}}$ remains positive, where the MagnetoHydroDynamic (MHD) contribution $\delta \hat{W}_{\mathrm{MHD}}$ usually is negative, and therefore destabilizing, while the kinetic contribution $\delta \hat{W}_{\text {kin }}$ can, under certain circumstances, be positive and therefore stabilizing. The MISHKA1 code $^{18}$ is used to retrieve $\delta \hat{W}_{\text {MHD }}$ for the studied sawteeth. $\delta \hat{W}_{\text {kin }}$ contains two parts: a part from the NBI-accelerated particles, here called $\delta \hat{W}_{\mathrm{NBI}}$, and a part from the ICRH-accelerated particles, here called $\delta \hat{W}_{\mathrm{ICRH}} \cdot \delta \hat{W}_{\mathrm{NBI}}$ is computed with an analytical expression, validated for JET in Ref. 6. The computation of $\delta \hat{W}_{\mathrm{ICRH}}$ is based on an integral formula from Ref. 9, and numerical values are obtained with the HAGIS ${ }^{19}$ code. The distribution of NBI-accelerated and ICRH-accelerated fast particles is obtained from the $\mathrm{SPOT}^{20}$ code, with input from the $\mathrm{NEMO}^{21}$ and $\mathrm{PION}^{22}$ codes, and run using the RFOF library. ${ }^{23}$
The effect of cyclotron current drive on sawteeth stabilization and destabilization has been studied in Ref. 24 . Though such effects may in general cases have a substantial impact on sawteeth, they are not expected to be significant in our experiments as dipole ICRH was used.

Besides, three of the four studied discharges (\#86762, \#86774 and \#86775) contained helium 3 (He3), in a proportion of 5 to $13 \%$. In those discharges, in addition to 3rd harmonic D heating, the ICRH may have led to some 2nd harmonic He3 heating. However, the power transferred to the plasma through 2nd harmonic He3 heating was found ${ }^{25}$ to be much lower than through 3rd harmonic $\mathrm{D}$ beam heating. For discharge \#86775, in the steadystate plasma $0.4 \mathrm{~s}$ after the ICRH power reached flat top, the ICRH power absorbed by the D beam was $84 \%$, while the power absorbed by He3 was $3 \%$ only, the remaining $13 \%$ being absorbed by electrons. The difference in the absorbed powers can be explained as follows: the 2nd harmonic He3 resonance layer occurs at higher magnetic field than the 3rd harmonic D resonance, which means that the ICRH waves first crosses the 3 rd harmonic D resonance before reaching the 2nd harmonic He3 resonance layer. In addition, the He3 ions were not pre-accelerated by NBI. Therefore, the effects of fast He3 particles have not been taken into account in the present work.

\section{BULK EQUILIBRIUM RECONSTRUCTION AND MHD EXCITATION OF INTERNAL KINK MODE}

For the bulk equilibrium reconstruction, the EFIT code has been run in conjunction with data from Motional Stark Effect (MSE) measurements. With this method, the accuracy of the $q$ profile is estimated ${ }^{26}$ to be of the order of 10-15\%. To generate a straight field line coordinate system required for MHD analysis, the HELENA $\operatorname{code}^{27}$ has then been used. The results are presented in table I.

The equilibrium computed by HELENA has been used as an input to the MISHKA1 code ${ }^{18}$ to compute the MHD growth rate of the internal kink mode for the four sawteeth studied, at different times, with accuracy estimated to be of the order of $3 \% .{ }^{16}$ This MHD growth rate has then been converted to the MHD potential energy functional $\delta \hat{W}_{\mathrm{MHD}}$ as described in Ref. 9:

$$
\delta \hat{W}_{\mathrm{MHD}}=-\frac{s_{1}}{2 \pi} \gamma \tau_{A}
$$

where $\gamma$ is the internal kink mode growth rate, $s_{1}=$ $\bar{r}_{1} q^{\prime}\left(\bar{r}_{1}\right)$ is the magnetic shear at the $q=1$ surface, $\bar{r}_{1}=r_{1} \sqrt{\kappa_{1}}$ is the average minor radius of the $q=1$ surface, $r_{1}$ is the minor radius of the $q=1$ surface on the equatorial plane, $\kappa_{1}$ is the ellipticity of the $q=1$ surface, $\tau_{A}=\sqrt{1+2 q^{2}} R_{0} / v_{A}=\sqrt{3} R_{0} / v_{A}$ is the Alfvén time, $R_{0}$ is the plasma major radius on the magnetic axis, $v_{A}=B_{0} / \sqrt{\mu_{0} n_{i} m_{i}}$ is the Alfvén speed, $B_{0}$ is the norm of the magnetic field on the magnetic axis, $\mu_{0}$ is the vacuum 


\begin{tabular}{lccc|ccc}
\hline \hline discharge & \multicolumn{3}{c}{86459} & \multicolumn{3}{c}{86762} \\
$\boldsymbol{t}[\mathbf{s}]$ & 10.66 & 10.79 & 10.99 & 10.52 & 10.79 & 11.04 \\
\hline $\boldsymbol{q}$ on axis & 0.96 & 0.93 & 0.87 & 0.97 & 0.88 & 0.85 \\
$\boldsymbol{R}(\boldsymbol{q}=\mathbf{1})[\mathbf{m}]$ & 3.33 & 3.35 & 3.40 & 3.29 & 3.38 & 3.38 \\
$\boldsymbol{s}_{\mathbf{1}}$ & 0.14 & 0.19 & 0.38 & 0.08 & 0.32 & 0.36 \\
$\boldsymbol{\delta} \hat{\boldsymbol{W}}_{\text {MHD }}\left[\mathbf{1 0}^{-\mathbf{3}}\right]$ & $\mathrm{N} / \mathrm{A}$ & -0.86 & -1.61 & $\mathrm{~N} / \mathrm{A}$ & -2.28 & -2.03 \\
\hline \hline
\end{tabular}

\begin{tabular}{lccc|ccc}
\hline \hline discharge & & 86774 & & 36775 \\
$\mathbf{t}[\mathbf{s}]$ & 9.33 & 9.47 & 9.63 & 10.46 & 11.12 & 11.35 \\
\hline $\boldsymbol{q}$ on axis & 0.99 & 0.95 & 0.85 & 0.75 & 0.71 & 0.69 \\
$\boldsymbol{R}(\boldsymbol{q}=\mathbf{1})[\mathbf{m}]$ & 3.33 & 3.36 & 3.43 & 3.42 & 3.42 & 3.43 \\
$\boldsymbol{s}_{\mathbf{1}}$ & 0.11 & 0.25 & 0.49 & 0.61 & 0.70 & 0.75 \\
$\boldsymbol{\delta} \hat{\boldsymbol{W}}_{\text {MHD }}\left[\mathbf{1 0}^{-\mathbf{3}}\right]$ & $\mathrm{N} / \mathrm{A}$ & -2.11 & -3.06 & -4.41 & -4.34 & -4.37 \\
\hline \hline
\end{tabular}

Table I. For the four studied sawteeth, four values are presented at different time slices: $q$ on axis, computed with HELENA; the radial position of the $q=1$ surface (equatorial plane, low field side), computed with HELENA; the magnetic shear on the $q=1$ surface $s_{1}$, computed with HELENA; and the MHD potential energy functional $\delta \hat{W}_{\mathrm{MHD}}$, computed with MISHKA1. The time slices here mentioned are indicated on Figure 1 with dashed lines.

magnetic permeability, $n_{i}$ is the peak ion density and $m_{i}$ is the average ion mass.

Table I presents the results from the MISHKA1 code computed for the four discharges when $\mathrm{q}(0)$ had a value equal to, or lower than 0.95 , corresponding to a strongly unstable kink mode.

\section{NBI CONTRIBUTION TO $\delta \hat{W}_{\text {kin }}$}

Let us recall that we have $\delta \hat{W}_{\text {kin }}=\delta \hat{W}_{\mathrm{NBI}}+\delta \hat{W}_{\mathrm{ICRH}}$. The NBI contribution reads: ${ }^{6}$

$$
\delta \hat{W}_{\mathrm{NBI}}=\frac{\sqrt{2} \mu_{0}}{2 \pi B_{0}^{2} \varepsilon_{1}^{1 / 2}}\left[\frac{3}{2} \int_{0}^{\rho_{1}} \frac{\rho^{1 / 2}}{\rho_{1}^{3 / 2}} p_{\mathrm{NBI}} d \rho-p_{\mathrm{NBI}}\left(\rho_{1}\right)\right],
$$

where $p_{\mathrm{NBI}}$ is the pressure of NBI-accelerated fast particles, and $\rho_{1}$ is the normalized radial position of the $q=1$ surface. It is found in Ref. 6 that this analytical expression gives, in the case of JET NBI, very good agreement with values obtained from numerical codes modelling kinetic-MHD modes interactions. The radial pressure profiles of the NBI-accelerated ions are obtained with the code SPOT $^{20}$ coupled with the NBI deposition simulation code NEMO. ${ }^{21}$ They are assumed to be stable during the time scales of interest, since the neutral particle injection was stable during the considered sawteeth, and since NBI fast particles are not expected to be affected by the tornado modes observed in discharges \#86459 and \#86762 (their energy is too low).

The results are presented on Figure 4 and discussed in Sec. VIII. Note that the NBI contribution $\delta \hat{W}_{\text {NBI }}$ is generally small compared to the ICRH contribution $\delta \hat{W}_{\text {ICRH }}$.

\section{ICRH-ACCELERATED FAST PARTICLES DISTRIBUTION}

The ICRH-accelerated ions are known to be mostly trapped, with their banana tips being at the ICRH resonant layer (situated in the central region, close to the magnetic axis in our experiments). Following Ref. 28, we here use an extension of the classical Stix model ${ }^{28,29}$ to high harmonics. In the central region, the radial variations of the background electron temperature and density are slow compared to the radial variations of the fast particle density. The following ansatz for the distribution function is thus employed:

$$
F_{k, \mathrm{ICRH}}=\lambda A(\rho) f_{E}(E) \delta\left(\Lambda-\Lambda_{0}\right),
$$

where $\lambda$ is a normalization factor, $\rho=r / \bar{a}$ is the normalized minor radius coordinate, $r$ is the minor radius coordinate, $\bar{a}=a \sqrt{\kappa_{a}}$ is the average minor radius of the plasma, $a$ is the minor radius of the plasma measured on the equatorial plane, $\kappa_{a}$ is the ellipticity of the plasma at $r=a, \Lambda=\mu_{k} B_{0} / E$ is the pitch coordinate, $E=1 / 2 m_{k} v^{2}$ is the kinetic energy, $m_{k}$ is the mass of the fast particles, $\mu_{k}=m_{k} v_{\perp}^{2} / 2 B$ is the magnetic moment, $v_{\perp}$ is the norm of the perpendicular velocity, $v$ is the total norm of the velocity, and $B$ is the norm of the magnetic field.

$\Lambda_{0}$ is the value of the pitch on the ICRH resonance layer. In the four discharges, the magnetic field was chosen to ensure that the resonance layer would be situated close to the magnetic axis: consequently $\Lambda_{0}$ is close to 1.0 in the four discharges. We have $\Lambda_{0}=1.04$ in discharge \#86459, $\Lambda_{0}=1.02$ in discharge \#86762, and $\Lambda_{0}=1.00$ in discharges \#86774 and \#86775. The influence of $\Lambda_{0}$ on sawtooth stabilization according to Porcelli's model is discussed in Sec. VIII.

The ICRH-accelerated fast particle distribution is modelled with the code $\mathrm{SPOT}^{20}$, with the use of the code $\mathrm{NEMO}^{21}$ (for the NBI deposition), the library RFOF ${ }^{23}$ (for the interaction between the ions and the ICRF wave) and the code PION ${ }^{22}$ (for the propagation of the ICRF wave in the plasma). The numerical results are presented on Figure 2, showing that a Gaussian-shape fit in the form

$$
A(\rho)=\rho^{\alpha} e^{-\left(\rho / \rho_{k}\right)^{2}}
$$

is a good approximation. Here, $\rho_{k}$ is the characteristic length of the radial distribution, and $\alpha$ is a constant used to ensure that the maximum of the distribution function is reached on the resonance layer.

The TOFOR diagnostic ${ }^{30}$ has been used to measure the energy distribution of the neutrons emitted by reactions with fast deuterium. From this energy distribution of the neutrons, it has been possible $e^{28,31-33}$ to deduce the energy distribution $f_{E}$ of the fast deuterium 

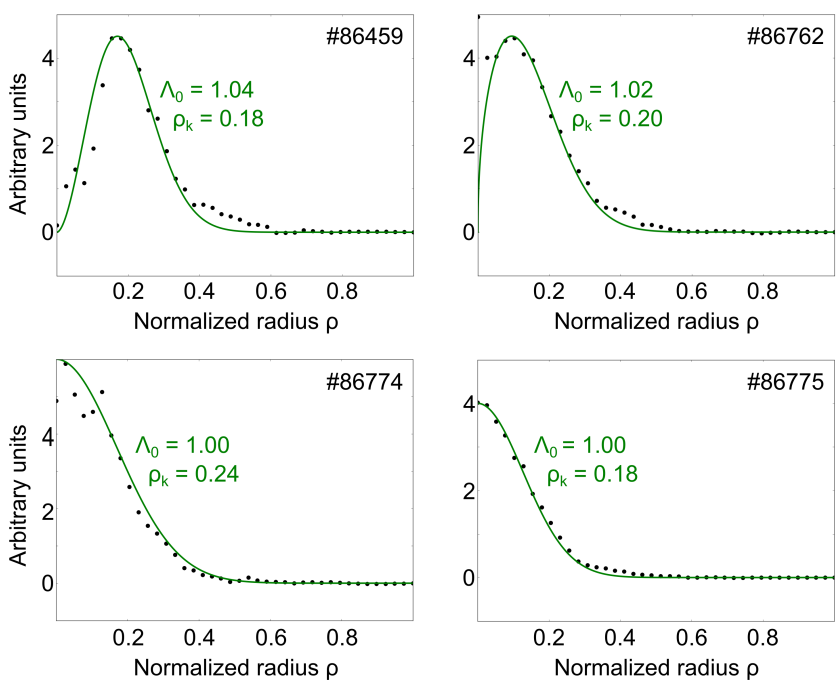

Figure 2. Radial fast ion distribution: data from SPOT (black circles) and corresponding fits (green, solid lines) - discharges \#86459, \#86762, \#86774 and \#86775.

\begin{tabular}{lcccc}
\hline \hline discharge & 86459 & 86762 & 86774 & 86775 \\
$\boldsymbol{W}_{\boldsymbol{k}, \text { ICRH }}[\mathrm{kJ}]$ & 322 & 252 & 249 & 615 \\
\hline \hline
\end{tabular}

Table II. $W_{k, \text { ICRH }}$ for the studied discharges: data from SPOT.

particles. The deduced energy distributions are shown on Figure 3, along with analytic distributions obtained by solving a Fokker-Planck equation derived in Ref. 29. More details about this Fokker-Planck modeling can be found in Ref. 28. On Figure 3, the cut-off energies in the analytic distributions have been adjusted so that the resulting neutron energy distribution be in agreement with the TOFOR measurements. It can be seen that the deuterium distributions are similar for the four studied sawteeth, with cut-off energies varying between 1.6 and $2.4 \mathrm{MeV}$.

Note that on Figures 2 and 3, the y-axis units are arbitrary; what matters is the relative profile of the distribution only: the normalization of the distribution functions is contained in $\lambda$ in expression (2), this $\lambda$ being determined below in Sec. VI.

\section{NORMALIZATION OF THE ICRH DISTRIBUTION FUNCTION}

In expression (2), $A$ and $f_{E}$ provide the profiles of the fast particle distribution function, but none of those functions yields the normalization of $F_{k, \mathrm{ICRH}}$. This normalization is represented by $\lambda$, a constant in space and velocity which is determined in this section for each studied discharge, using the total energy of the ICRH-accelerated fast particles $W_{k, \mathrm{ICRH}}$. It is necessary to determine $\lambda$ for the computation of $\delta \hat{W}_{\mathrm{ICRH}}$ (see Sec. VII). $W_{k, \mathrm{ICRH}}$ is defined as

$$
W_{k, \mathrm{ICRH}}=\int d r^{3} d v^{3} E F_{k, \mathrm{ICRH}}
$$

Taking into account expression (2) of $F_{k, I C R H}$ and the expressions of $A$ and $f_{E},(4)$ can be written, at lowest order in the inverse aspect ratio $\varepsilon=r / R_{0}$, as

$W_{k, \mathrm{ICRH}}=\frac{8 \pi^{2} R_{0} \bar{a}^{2}}{m \sqrt{2 m}} \int_{\rho_{\text {min }}}^{1} d \rho \int_{-\theta_{t}}^{\theta_{t}} d \theta \int_{0}^{+\infty} d E \frac{\lambda \rho^{\alpha+1} e^{-\left(\rho / \rho_{k}\right)^{2}} f_{E}(E) E^{3 / 2}}{\sqrt{1-\Lambda_{0}+\Lambda_{0} \varepsilon \cos \theta}}$,

where $\theta$ is the poloidal angle counted from the equatorial plane and $\rho_{\min }$ is the minimum value of $\rho$ reached by fast particles.

Defining $c_{r \theta}=\int_{\rho_{\text {min }}}^{1} \int_{-\theta_{t}}^{\theta_{t}} \frac{\rho^{\alpha+1} e^{-\left(\rho / \rho_{k}\right)^{2}}}{\sqrt{1-\Lambda_{0}+\Lambda_{0} \varepsilon \cos \theta}} d \theta d \rho$ and $c_{E}=\int_{0}^{+\infty} E^{3 / 2} f_{E}(E) d E, W_{k, \mathrm{ICRH}}$ reads

$$
W_{k, \mathrm{ICRH}}=\frac{8 \pi^{2} \lambda R_{0} \bar{a}^{2} c_{r \theta} c_{E}}{m_{k} \sqrt{2 m_{k}}}
$$

The numerical values of $W_{k, \text { ICRH }}$ have been retrieved from SPOT results; they are indicated in Table II. In discharges 86459,86762 and 86775 , the ICRH power was stable during the studied sawteeth; therefore $W_{k, \mathrm{ICRH}}$ can be considered as stable over time. In discharge 86774 , the studied sawtooth occurred during a power ramp-up: this ramp-up was not taken into account in SPOT, a time-averaged power value being used instead. However, the rationale presented in Sec. VIII is not altered by this fact: what matters is the evolution of $\delta \hat{W}_{\text {kin }}$ in time, which is found to increase. Taking into account the ICRH power ramp-up in discharge 86774 would only accentuate the increase of $\delta \hat{W}_{\text {kin }}$ over time, and would thus not bring any significant change to the global picture.

\section{ICRH CONTRIBUTION TO $\delta \hat{W}_{\text {kin }}$}

\section{A. Expression of $\delta \hat{W}_{\mathrm{ICRH}}$}

To compute $\delta \hat{W}_{\text {ICRH }}$, an integral expression, equation (11) of Ref. 9, is used. This expression is valid in the following limit: when the parallel pressure of fast particles is small compared to the perpendicular pressure of fast particles, and when the internal kink mode frequency is small compared to the banana drift frequency (condition (2) in Ref. 9). This limit is relevant to our experiment for the ICRH-accelerated particles, which are in the $\mathrm{MeV}$ range (see TOFOR results in Sec. V).

We thus have, with SI units, 

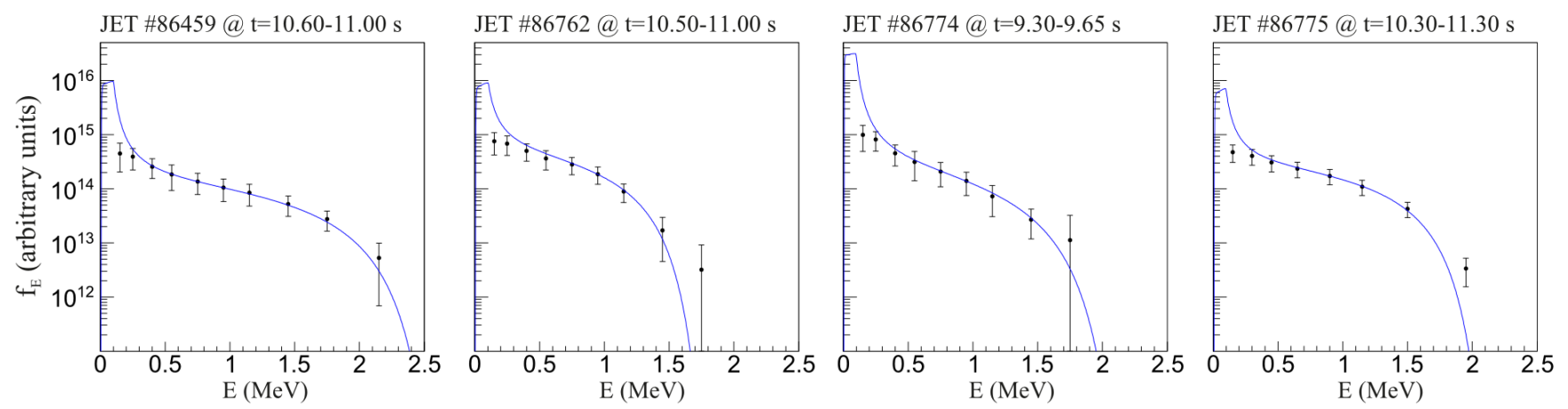

Figure 3. Energy distribution retrieved from TOFOR measurements (circle marks with error bars) and analytic fit (full line curve) - discharges \#86459, \#86762, \#86774 and \#86775.

$$
\delta \hat{W}_{I C R H}=\frac{\pi \mu_{0} \varepsilon_{1}}{2 B_{p}^{2}\left(\bar{r}_{1}\right) \bar{r}_{1}}\left(\frac{2}{m_{k}}\right)^{3 / 2} \int_{0}^{\bar{r}_{1}} d r r \int_{1-\varepsilon}^{1+\varepsilon} d \Lambda \Lambda I_{b} \frac{I_{c} I_{d}-I_{q}^{2}}{I_{d}} \int_{0}^{+\infty} d E E^{3 / 2} \frac{\partial F_{k, \mathrm{ICRH}}}{\partial r}
$$

where $\varepsilon_{1}=\bar{r}_{1} / R_{0}$ is the inverse aspect ratio at the $q=1$ surface, $B_{p}\left(\bar{r}_{1}\right)=\bar{r}_{1} B_{0} / R_{0}$ is the poloidal component of the magnetic field on the $q=1$ surface, $I_{b}=\frac{v \tau_{b}}{R q}$, $I_{c}=\langle\cos \theta\rangle_{b}, I_{d}=\langle\cos \theta\rangle_{b}+s\langle\theta \sin \theta\rangle_{b}, I_{q}=\langle\cos (q \theta)\rangle_{b}$, $\langle\cdot\rangle_{b}$ represents the average over the bounce motion, $v=$ $\sqrt{2 E / m_{k}}$ is the velocity of the particle, and $\tau_{b}$ is the bounce period.

Taking into account the expression of $F_{k, \text { ICRH }}$, we have

$$
\frac{\partial F_{k, \mathrm{ICRH}}}{\partial r}=\frac{\lambda}{\bar{a}}\left(\frac{\alpha}{\rho}-\frac{2 \rho}{\rho_{k}^{2}}\right) \rho^{\alpha} e^{-\left(\rho / \rho_{k}\right)^{2}} \delta\left(\Lambda-\Lambda_{0}\right) f_{E}(E)
$$

Using equation (5) to replace $\lambda$ in this expression, $\delta \hat{W}_{\text {ICRH }}$ reads

$$
\delta \hat{W}_{\mathrm{ICRH}}=\frac{\mu_{0} W_{k}}{4 \pi c_{r \theta} \bar{a} \bar{r}_{1}^{2} B_{0}^{2}} \int_{\rho_{\text {min }}}^{\rho_{1}} d \rho \rho^{\alpha+1}\left(\frac{\alpha}{\rho}-\frac{2 \rho}{\rho_{k}^{2}}\right) e^{-\left(\rho / \rho_{k}\right)^{2}} I\left(\Lambda_{0}\right),
$$

where $I\left(\Lambda_{0}\right)$ is defined as $\Lambda I_{b} \frac{I_{c} I_{d}-I_{q}^{2}}{I_{d}}$ evaluated at $\Lambda=$ $\Lambda_{0}$.

\section{B. Computation of $I\left(\Lambda_{0}\right)$ with HAGIS}

The HAGIS code ${ }^{19}$ is used to compute the orbit of fast test particles; ellipticity and Finite Orbit Width (FOW) effects are accounted for. From those orbits, $I\left(\Lambda_{0}\right)$ is computed numerically. The output of HELENA is used for the safety factor and the magnetic shear radial profiles; those profiles are used to numerically compute the integral over $\rho$ in expression (7). The results are presented on Figure 4 and discussed in Sec. VIII.

\section{PREDICTIONS OF THE MODEL: SAWTEETH STABILIZATION}

The evolutions of $\delta \hat{W}=\delta \hat{W}_{\mathrm{MHD}}+\delta \hat{W}_{\text {kin }}$ are shown on Figures $4 \mathrm{a}$ ) and $4 \mathrm{~b}$ ). According to Porcelli's theory, a sawtooth crash is due to occur when $\delta \hat{W}$ becomes negative.

In the case of discharges \#86459 and \#86762, the dashed lines show the evolution of $\delta \hat{W}$ assuming $\rho_{k}$ (characteristic length of the ICRH fast particles radial distribution) remains constant. This evolution is unlikely, considering the tornado modes which occurred during those two discharges (see Sec. IX A below). The full lines show the probable evolution of $\delta \hat{W}$, assuming $\rho_{k}$ increases during the tornado modes activity. On Figure $4 \mathrm{a}$ ), an increase of 0.05 of $\rho_{k}$ has been retained, consistent with the observations made in a JET discharge exhibiting similar features (Ref. 16). In discharges \#86459 and \#86762, gamma-rays signals were too low to retrieve any useful data, and neutron cameras were not in activity, thus prohibiting the direct experimental observation of the fast particle radial redistribution in discharges \#86459 and \#86762. Note that the situation was different during the sawtooth analysed in Ref. 16: discharge \#74951 was performed in JET with carbon (C) wall, thus allowing gamma-rays from $\mathrm{D}$ (fast) $+\mathrm{C}$ nuclear reactions to be analysed. In our recent four discharges, the metallic ITER-like wall (beryllium and tungsten) did not give us such opportunity.

In the case of discharges \#86774 and \#86775, neutron cameras $^{34}$ with horizontal and vertical lines of sight were available (see Figure 5). Thanks to the reaction $\mathrm{D}_{\text {fast }}+\mathrm{D}_{\mathrm{th}} \rightarrow n+{ }^{3} \mathrm{He}$, a broadening of the radial distribution of the fast deuterons during the sawteeth 


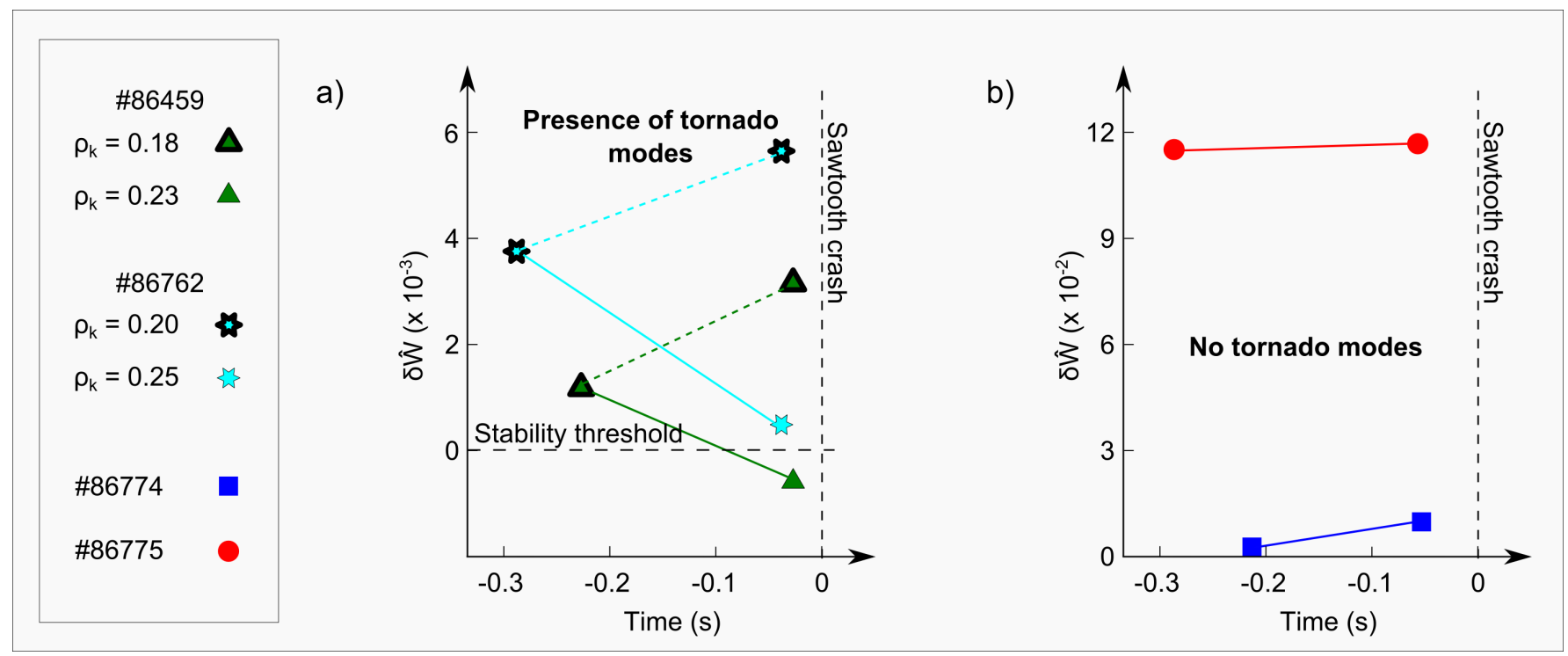

Figure 4. Evolutions of $\delta \hat{W}=\delta \hat{W}_{\mathrm{MHD}}+\delta \hat{W}_{\text {kin }}$ in the case of discharges \#86459 and \#86762 (figure a), as well as discharges \#86774 and \#86775 (figure b), as functions of time before the sawtooth crashes. For discharges \#86459 and \#86762, different values of $\rho_{k}$ have been assumed: the dashed lines show the evolution of $\delta \hat{W}$ for stationary values of $\rho_{k}$; the full lines show the probable evolution of $\delta \hat{W}$ assuming $\rho_{k}$ increases in time. For discharges \#86774 and \#86775, the full lines show the evolution of $\delta \hat{W}$ taking into account the stability of $\rho_{k}$ as indicated by the timelines from neutron cameras.

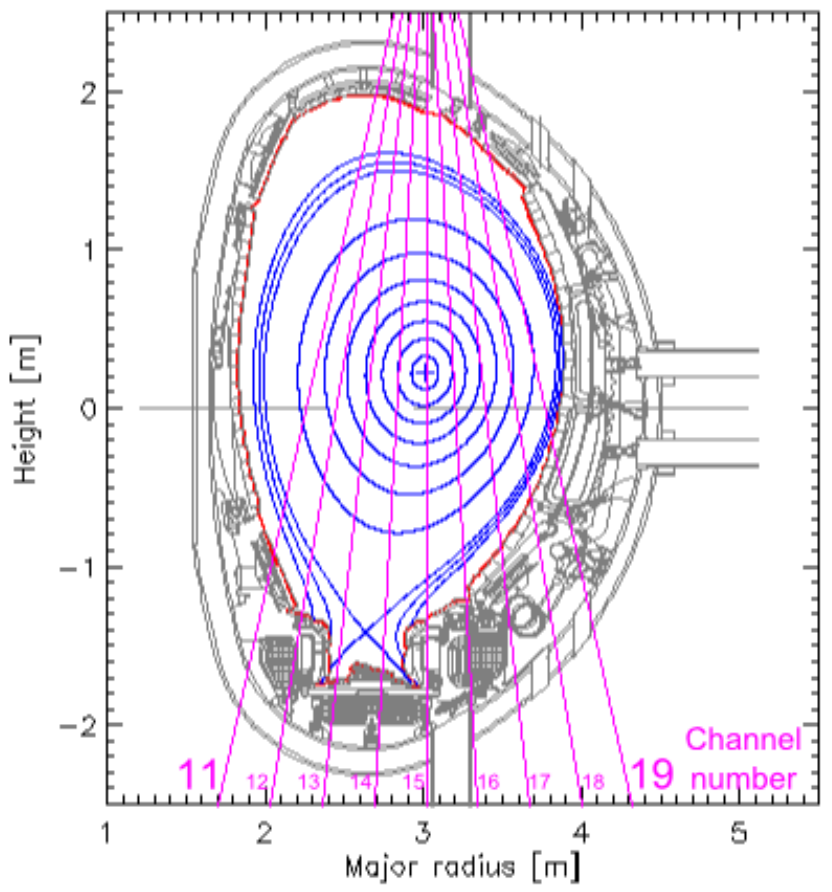

Figure 5. Lines of sight of vertical neutron cameras in JET. The blue circles correspond to the magnetic flux surfaces computed with EFIT in discharge 86775 , at time $\mathrm{t}=10.96 \mathrm{~s}$.

would have been observable on the neutron cameras signals (the signals from the outer channels would have increased while the signals from the inner channels would have decreased, like in Ref. 16). However, in discharges \#86774 and \#86775, no significant evolution
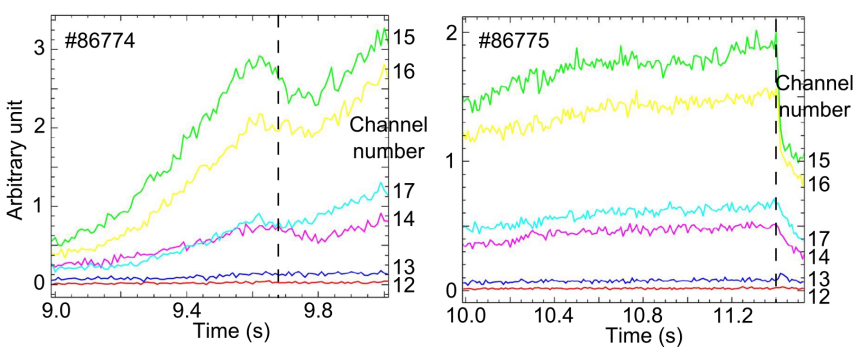

Figure 6. Time lines from neutron cameras, vertical channels 12 to 17, discharges \#86774 and \#86775. The dashed lines show the times of the sawtooth crashes.

of the neutron cameras signals was observed during the studied sawteeth (see Figure 6), which means that the fast particles were not expelled from within the $q=1$ surface. In partcular, in the case of discharge \#86775, the fast particle population was remarkably stable. This is consistent with the fact that no tornado modes were observed (see Sec. IX A below). Consequently, the time evolution of $\delta \hat{W}$ has been plotted at constant $\rho_{k}$ for those two discharges.

It can be observed on Figure $4 \mathrm{~b}$ ) that $\delta \hat{W}$ does not get closer to the crash threshold $(\delta \hat{W}=0)$ when time goes by. On the contrary, $\delta \hat{W}$ tends to get further from the threshold. This fact can be interpreted considering that the fast particle distribution features $\left(\Lambda_{0}, \rho_{k}\right.$, $\left.W_{k, \text { ICRH }}\right)$ remain the same, while the radial position of the $q=1$ surface increases (see table I) and $q$ on axis decreases. Consequently, the fast particles are found to be contained deeper and deeper within the $q=1$ surface 
as time increases, and they are more stabilizing. ${ }^{9,10}$ The evolutions of $\delta \hat{W}$ computed for discharges \#86774 and \#86775 show that the sawtooth crashes for those discharges were caused by events which are fast (a few $10 \mathrm{~ms}$ ) compared to the sawtooth characteristic period, since no evolution of $\delta \hat{W}$ in the direction of a crash is observed during the sawtooth.

It can be observed on Figure 4 a) that $\delta \hat{W}$ is highly sensitive to the value of $\rho_{k}$ : this is consistent with the theory. ${ }^{9,10}$ In discharges \#86459 and \#86762, without any increase of $\rho_{k}$, Porcelli's model would not predict the sawtooth crash, since $\delta \hat{W}$ then increases over time and gets further from the crash threshold. On the contrary, taking into account an increase of $\rho_{k}$ is consistent with the happening of the crash, in the framework of Porcelli's model.

The absolute values of $\delta \hat{W}$ depend strongly not only on $\rho_{k}$ but also on $\Lambda_{0}$. The closer $\Lambda_{0}$ is to 1.0, the more stabilizing the fast particles are. This is consistent with the fact that when $\Lambda_{0}=1.0$, the fast particles are contained deeper within the $q=1$ surface and are therefore more stabilizing. It may be difficult to determine $\Lambda_{0}$ with the accuracy necessary for the computation of $\delta \hat{W}$ : the needed accuracy is of the order of $1 \%$ for the value of $B_{0}$ (magnetic field on axis), which is not met by EFIT with MSE. However, contrary to $\rho_{k}, \Lambda_{0}$ is expected to remain stable over times of a few hundreds of milliseconds, and the time evolution of $\delta \hat{W}$ is hardly sensitive to the value retained for $\Lambda_{0}$. This means that even if the original error made on $\Lambda_{0}$ is higher than $1 \%$, this does not affect the rationale here exposed: what really matters is that $\Lambda_{0}$ is stable during the considered time scales, and that $\delta \hat{W}$ increases over time if $\rho_{k}$ remains constant.

It can finally be observed on Figure 4 that $\delta \hat{W}$ is much higher in the case of discharge \#86775 than in the three other cases. This is consistent with the fact that the corresponding sawtooth was much longer (over $2.5 \mathrm{~s}$ ) than the three other studied sawteeth. This high value of $\delta \hat{W}$ can be explained by the high value of the fast particle energy (see Table II) and by the favourable radial position of the fast particles $\left(\Lambda_{0}=1\right.$ and small $\rho_{k}$ : see Figure 2) in the case of discharge \#86775.

\section{EXPLANATION FOR THE CRASHES}

\section{A. Tornado modes}

Tornado modes ${ }^{35,36}$ are observed in discharges \#86459 and \#86762 with Far InfraRed (FIR) interferometry (Figures 7 and 8). Those tornado modes have an overall duration of more than $0.2 \mathrm{~s}$ before the sawtooth crashes. Tornado modes are known to be at the origin of the expulsion of some fast particles from within the $q=1$

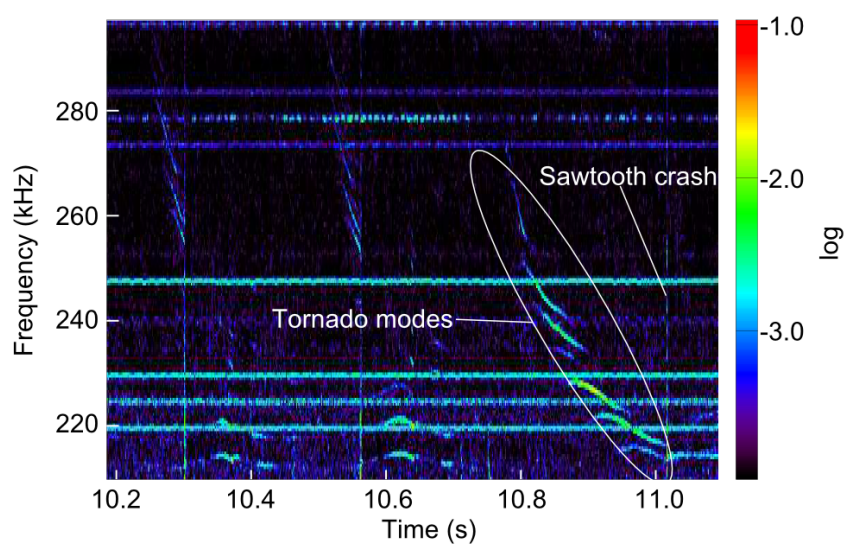

Figure 7. Tornado modes measured with FIR interferometry - discharge \#86459.

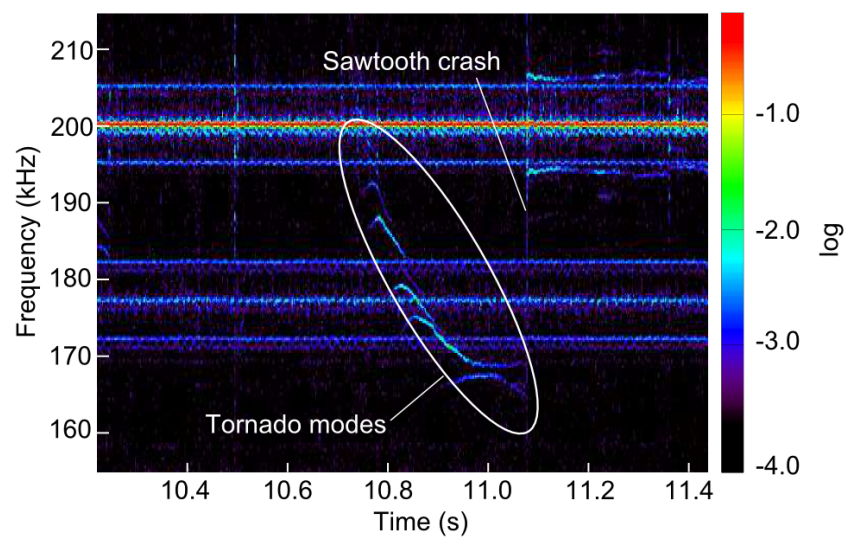

Figure 8. Tornado modes measured with FIR interferometry - discharge \#86762.

surface, as has been observed in JT-60, ${ }^{35}$ TFTR, ${ }^{37}$ DIII$\mathrm{D},{ }^{38}$ and JET. ${ }^{16}$ In particular, the JET discharge studied in Ref. 16 was very similar to the discharges considered in the present study (same magnetic field on axis, same plasma current, same 3rd harmonic D ICRH). Such expulsion of fast particles during the sawteeth means that the stabilizing effect of the fast particles is strongly reduced; this can lead to the crash of the sawteeth, as exposed in Ref. 16, 37, and 38. In our experiments, the expulsion of the fast particles outside the $q=1$ surface can be modelled as the increase of $\rho_{k}$ during the sawtooth periods. Porcelli's model then shows that this expulsion of fast particles is consistent with the crashes and sufficient to account for them: see Section VIII and Figure 4 a).

In the case of discharges \#86774 and \#86775, no tornado modes were observed. One can notice that the two discharges exhibiting tornado modes were the two with $\Lambda_{0}>1.0$, while the two-discharges with $\Lambda_{0}=1.0 \mathrm{did}$ not exhibit tornado modes. 

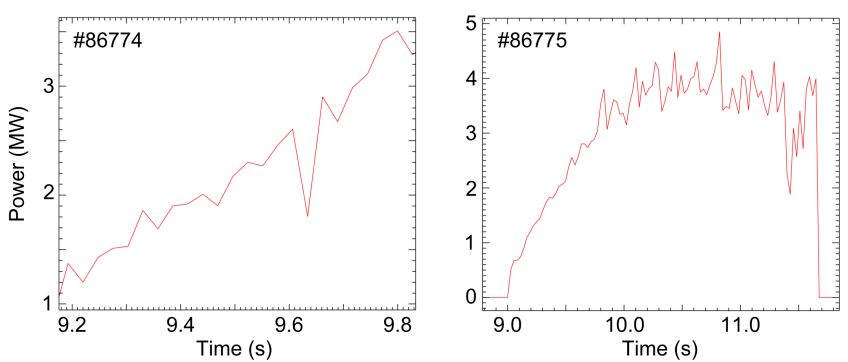

Figure 9. ICRH coupled power - discharges \#86774 and \#86775.
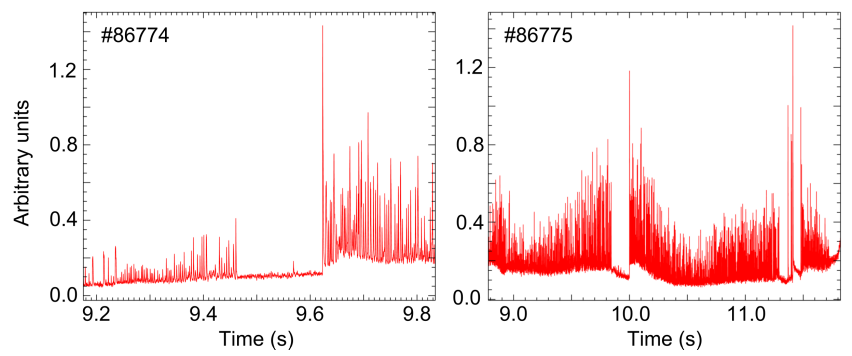

Figure 10. ELMs (D alpha) - discharges \#86774 and \#86775.

\section{B. ELMs - Inward propagation of a cold front}

In discharges \#86774 and \#86775, as can be seen on Figure 9, significant drops in the radio-frequency $(\mathrm{RF})$ coupled power are observed just before the sawtooth crashes: a drop of $31 \%$ in the case of discharge \#86774, and a drop of $43 \%$ in the case of discharge \#86775. Could those power drops be at the origin of a decrease of the stabilizing fast particle population within the $q=1$ surface, thus leading to the observed crashes? The slowing down time for the fast particle population can be estimated with the Spitzer time $\tau_{s}:{ }^{39}$

$$
\tau_{s}=\frac{3(2 \pi)^{3 / 2} T_{e}^{3 / 2} \varepsilon_{0}^{2} m_{k}}{n_{e} \sqrt{m_{e}} e^{4} \ln \Lambda}
$$

where $T_{e}$ is the electron temperature, $\varepsilon_{0}$ the vacuum permittivity constant, $m_{e}$ is the electron mass, $e$ the elementary Coulomb charge and $\ln \Lambda$ is the Coulomb logarithm.
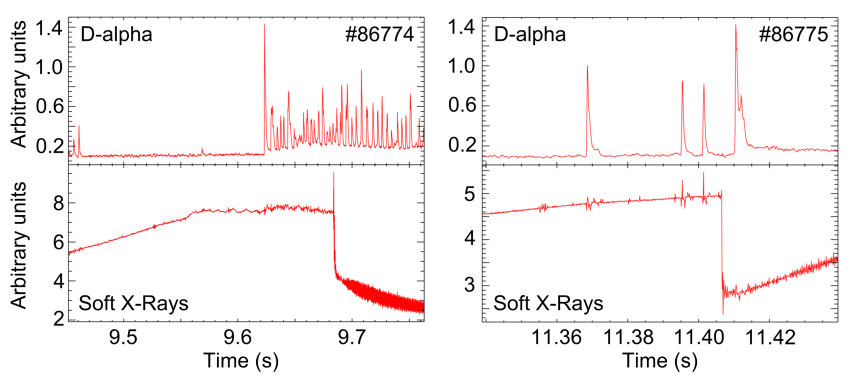

Figure 11. ELMs (D alpha) and Soft X-Rays - discharges \#86774 and \#86775.
With the set of parameters corresponding to the four discharges here considered, $\ln \Lambda$ reads: ${ }^{40}$

$$
\ln \Lambda=24-\ln \left(\frac{\sqrt{n_{e}}}{T_{e}}\right) .
$$

For discharge \#86774, expression (8) yields $\tau_{s}=0.6 \mathrm{~s}$, while it yields $\tau_{s}=0.9 \mathrm{~s}$ for discharge \#86775. In comparison, the times between the RF power drops and the sawtooth crashes are one order of magnitude lower: it is comprised between 0.04 and 0.08 s for discharge \#86774, while it is comprised between 0.005 and $0.04 \mathrm{~s}$ for discharge \#86775. Those intervals correspond to the time intervals between two measurements of the RF power.

Another hypothesis is that the RF power drops have been caused by ELM bursts, and that in addition to causing the RF power drops, those ELM bursts have also triggered the sawtooth crashes. The measured activity of D-alpha transition is represented on Figure 10 for discharges \#86774 and \#86775 during the studied sawteeth.

In the case of discharge \#86774, one major ELM burst occurs at $t=9.62 \mathrm{~s}$, while no other significant burst occurs before. This burst takes place about $60 \mathrm{~ms}$ before the sawtooth crash, as can be seen on Figure 11 where D-alpha activity and soft X-ray signals are represented together, with a focus on the crash time.

In the case of discharge \#86775, two periods of major ELM activity are recorded: one around $t=10.0 \mathrm{~s}$, which does not have any significant impact on the coupled ICRH power, and one around $t=11.4 \mathrm{~s}$, at the same time as the sawtooth crash. It can be seen on Figure 11 that in the second period of ELM activity, the first burst occurs about $40 \mathrm{~ms}$ before the sawtooth crash. That the first period of ELM activity should have had no impact on the coupled ICRH power makes it likely that the intensity of ELMS was then weaker than during the second period of activity; and this is consistent with the fact that it should not have triggered any sawtooth crash either. It is also consistent with the fact that during the first period of ELM activity $(t=10.0 \mathrm{~s}), \delta \hat{W}_{\mathrm{MHD}}$ computed with MISHKA1 was about twice as low as during the second period ( $t=11.4 \mathrm{~s}$ ), thus indicating that the internal kink mode was not as easy to be destabilized during the first period of ELM activity as during the second period.

It has been reported in Ref. 41 that some large ELM bursts can generate a strong perturbation $\delta T_{e}$ which propagates inwards at a velocity of the order of a few $100 \mathrm{~m} \cdot \mathrm{s}^{-1}$. In the two discharges of interest, the $q=1$ surface is situated quite far from the magnetic axis, at $R=3.43 \mathrm{~m}$ on the equatorial plane, low field side (see table I). Thus, the $\delta T_{e}$ perturbation can reach this surface after travelling about $50 \mathrm{~cm}$, which correspond to less than $5 \mathrm{~ms}$. This time value is lower than the time which elapses between the first ELM burst of interest and the sawtooth crash in both discharges \#86774 (60 ms) and \#86775 (40 ms), which makes it possible for the ELM bursts to be at the origin of the sawtooth crashes.

Figures 12 and 13 show the time evolution of the electron temperature in discharges \#86774 and \#86775 mea- 


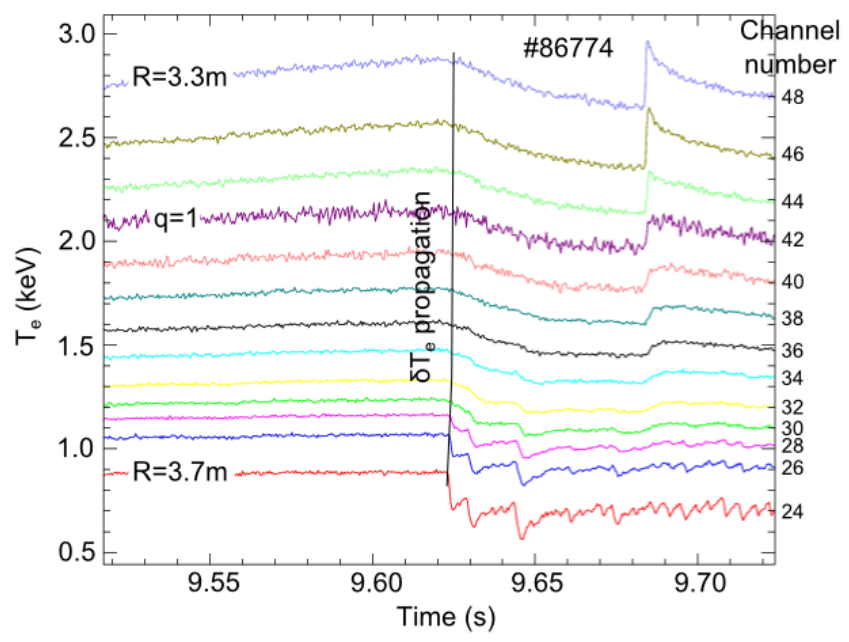

Figure 12. Time evolution of the electron temperature in discharge \#86774 measured with various ECE channels, corresponding to various radial positions comprised between $R=3.3 \mathrm{~m}$ (channel 48 ) and $R=3.7 \mathrm{~m}$ (channel 24). The $q=1$ surface corresponds approximately to channel 42 .

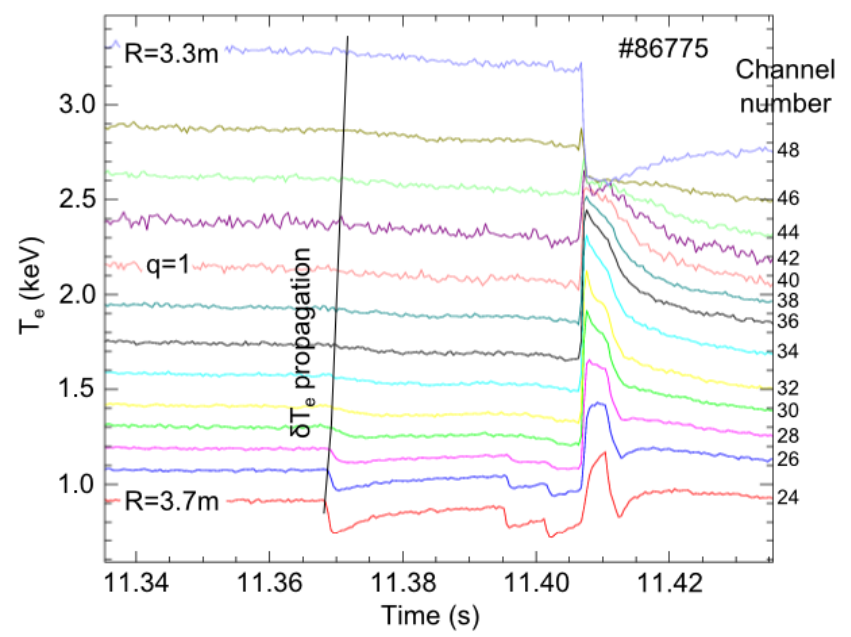

Figure 13. Time evolution of the electron temperature in discharge \#86775 measured with various ECE channels, corresponding to various radial positions comprised between $R=3.3 \mathrm{~m}$ (channel 48 ) and $R=3.7 \mathrm{~m}$ (channel 24). The $q=1$ surface corresponds approximately to channel 40 .

sured with various ECE channels, corresponding to various radial positions comprised between $R=3.3 \mathrm{~m}$ (channel 48) and $R=3.7 \mathrm{~m}$ (channel 24). On those two figures, the propagation of the cold front ( $\delta T_{e}$ perturbation) can be seen and is identified with a solid line. In the case of discharge \#86775, the cold front does not appear on figure 13 clearly enough for immediate identification; this is why larger scale plots have been represented on Figure 14. Note that on that last figure, only the vertical scale (corresponding to the electron temperature) has been increased with respect to Figure 13, while the $\mathrm{x}$-axis scale has been kept similar to that of Figure 13.

The inwards propagating perturbation triggered by
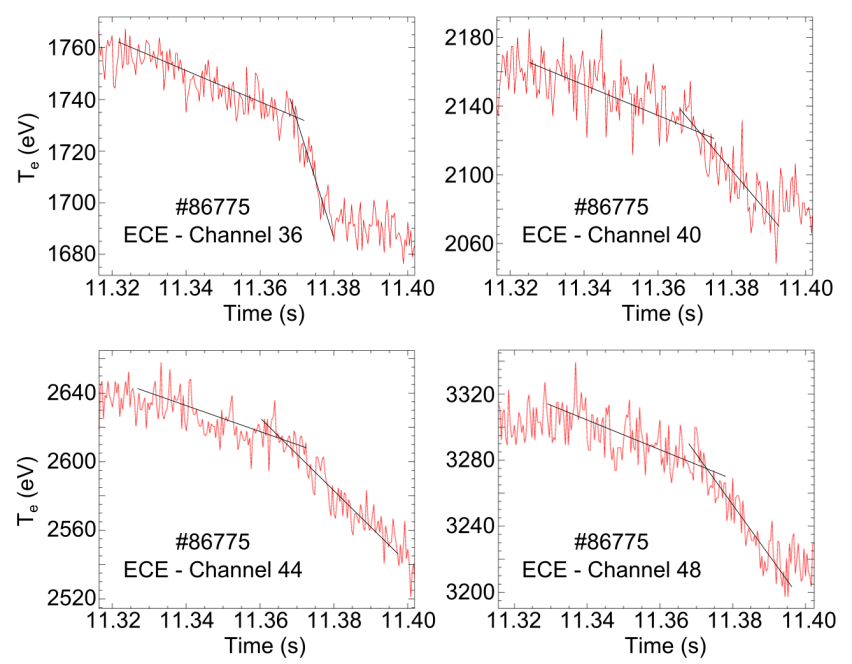

Figure 14. Details of the time evolution of the electron temperature in discharge \#86775 measured by four different ECE channels (channels 36, 40, 44 and 48), showing the propagation of the cold front to the $q=1$ surface (corresponding approximately to channel 40) and beyond (channels 44 and 48).

ELM bursts was measured in Ref. 41 on $T_{e}$ and is here measured experimentally on $T_{e}$ as well. However other plasma parameters are likely to be affected by the inward propagating front, and be perturbed at the same time as the electron temperature. The mechanism of sawtooth destabilization by such perturbations triggered by ELM bursts is not clear yet. Those perturbations may have an impact on the $q=1$ layer, and in particular on the magnetic shear $s_{1}$ and the diamagnetic frequency $\omega_{*}$; but this hypothesis has not been confirmed and the exact mechanism remains to be determined.

\section{CONCLUSION}

Four sawteeth in four different JET discharges have been analysed. The application of Porcelli's model to those sawteeth, with the help of the equilibrium codes EFIT and HELENA, of the MHD code MISHKA1, of the fast-particle codes SPOT and HAGIS has enabled us to check that fast particles produced by NBI and 3rd harmonic D ICRH had a strong stabilizing effect on the internal kink mode. Despite the strong stabilization thus gained, the four sawtooth all ended up crashing. Two mechanisms that can explain those crashes have been observed.

The first one is the exhibition of tornado modes before the sawtooth crashes in discharges \#86459 and \#86762. Tornado modes are known ${ }^{16,35,37,38}$ to expel fast particles from within the $q=1$ surface, leading to the loss of their stabilizing effect. In such situations, fast particles are not only the cause of the sawtooth stabilization, they are also the cause of the sawtooth crashes, since they are at 
the origin of the tornado modes which eventually trigger the sawtooth crashes.

The second one is an inward propagating perturbation $\delta T_{e}$ of the electron temperature, triggered by major ELM bursts. The inward propagation of the perturbation occurs at ballistic velocities ${ }^{41}\left(\sim 160 \mathrm{~m} \cdot \mathrm{s}^{-1}\right)$ : the time of the propagation from the edge of the plasma to the $q=1$ surface is therefore under $5 \mathrm{~ms}$ in JET. This phenomenon is believed to be at the origin of the sawtooth crashes in discharges \#86774 and \#86775. An implication of this hypothesis is that the cause for sawtooth crashes comes from outside the $q=1$ surface, and not from the very fast particles which provide stabilization.

A limit of the present study is that the possible mechanism of the interaction between the cold front in discharges \#86774 and \#86775 and the internal kink mode has not been analysed. Further theoretical end experimental analyses are required for that.

\section{ACKNOWLEDGMENTS}

This work has been carried out within the framework of the EUROfusion Consortium, has received funding from the Euratom research and training programme 20142018 under grant agreement No 633053, and was partfunded by the RCUK Energy Programme [grant number EP/I501045]. The views and opinions expressed herein do not necessarily reflect those of the European Commission.

The authors would like to thank Torbjörn Hellsten for fruitful discussion.

\section{REFERENCES}

${ }^{1}$ A. Y. Aydemir, Physics of Fluids B: Plasma Physics (1989-1993) 4, 3469 (1992).

${ }^{2}$ M. N. Bussac, R. Pellat, D. Edery, and J. L. Soule, Phys. Rev. Lett. 35, 1638 (1975).

${ }^{3}$ B. B. Kadomstev, Sov. J. Plasma. Phys. 1, 389 (1975).

${ }^{4}$ F. L. Waelbroeck, Physics of Fluids B: Plasma Physics (19891993) 1, 2372 (1989).

${ }^{5}$ L. Zakharov, B. Rogers, and S. Migliuolo, Physics of Fluids B: Plasma Physics (1989-1993) 5, 2498 (1993).

${ }^{6}$ C. Angioni, A. Pochelon, N. N. Gorelenkov, K. G. McClements, O. Sauter, R. V. Budny, P. C. de Vries, D. F. Howell, M. Mantsinen, M. F. F. Nave, S. E. Sharapov, and contributors to the EFDA-JET Workprogramme, Plasma Physics and Controlled Fusion 44, 205 (2002).

${ }^{7}$ D. Edery, X. Garbet, J. P. Roubin, and A. Samain, Plasma Physics and Controlled Fusion 34, 1089 (1992).

${ }^{8}$ J. P. Graves, Physics of Plasmas (1994-present) 12, 090908 (2005).

${ }^{9}$ F. Porcelli, Plasma Physics and Controlled Fusion 33, 1601 (1991).

${ }^{10}$ F. Porcelli, R. Stankiewicz, H. L. Berk, and Y. Z. Zhang, Physics of Fluids B: Plasma Physics (1989-1993) 4, 3017 (1992).

${ }^{11}$ F. Porcelli, D. Boucher, and M. N. Rosenbluth, Plasma Physics and Controlled Fusion 38, 2163 (1996).

${ }^{12}$ R. B. White, P. H. Rutherford, P. Colestock, and M. N. Bussac, Phys. Rev. Lett. 60, 2038 (1988).
${ }^{13}$ R. B. White, M. N. Bussac, and F. Romanelli, Phys. Rev. Lett. 62, 539 (1989).

${ }^{14}$ M. Zabiego, X. Garbet, A. Becoulet, F. Nguyen, and B. Saoutic, Nuclear Fusion 34, 1489 (1994).

${ }^{15}$ J. P. Graves, I. T. Chapman, S. Coda, M. Lennholm, M. Albergante, and M. Jucker, Nature Communications 3, 624 (2012).

${ }^{16}$ T. Gassner, K. Schoepf, S. E. Sharapov, V. G. Kiptily, S. D. Pinches, C. Hellesen, J. Eriksson, and JET-EFDA contributors, Physics of Plasmas (1994-present) 19, 032115 (2012), http://dx.doi.org/10.1063/1.3696858.

${ }^{17}$ G. F. Matthews, M. Beurskens, S. Brezinsek, M. Groth, E. Joffrin, A. Loving, M. Kear, M.-L. Mayoral, R. Neu, P. Prior, V. Riccardo, F. Rimini, M. Rubel, G. Sips, E. Villedieu, P. de Vries, M. L. Watkins, and EFDA-JET contributors, Physica Scripta 2011, 014001 (2011).

${ }^{18}$ A. B. Mikhailovskii, G. T. A. Huysmans, W. O. K. Kerner, and S. E. Sharapov, Plasma Physics Reports 23, 844 (1997).

${ }^{19}$ S. D. Pinches, L. C. Appel, J. Candy, S. E. Sharapov, H. L. Berk, D. Borba, B. N. Breizman, T. C. Hender, K. I. Hopcraft, G. T. A. Huysmans, and W. Kerner, Computer Physics Communications 111, 133 (1998).

${ }^{20}$ M. Schneider, L.-G. Eriksson, V. Basiuk, and F. Imbeaux, Plasma Physics and Controlled Fusion 47, 2087 (2005).

${ }^{21}$ M. Schneider, L.-G. Eriksson, I. Jenkins, J. Artaud, V. Basiuk, F. Imbeaux, T. Oikawa, JET-EFDA contributors, and ITM-TF contributors, Nuclear Fusion 51, 063019 (2011).

${ }^{22}$ L.-G. Eriksson, T. Hellsten, and U. Willen, Nuclear Fusion 33, 1037 (1993)

${ }^{23}$ T. Johnson, A. Salmi, G. Steinbrecher, L.-G. Eriksson, T. Hellsten, L. J. Höök, M. Schneider, and ITM-TF contributors, AIP Conference Proceedings 1406, 373 (2011).

${ }^{24}$ J. P. Graves, I. T. Chapman, S. Coda, T. Johnson, M. Lennholm, J. I. Paley, O. Sauter, and JET-EFDA Contributors, Fusion Science and Technology 59, 539 (2011).

${ }^{25}$ T. Hellsten, T. Johnson, S. Sharapov, V. Kiptily, J. Eriksson, M. Mantsinen, M. Schneider, F. Rimini, M. Tsalas, and JET contributors, 21st Topical Conference on Radiofrequency Power in Plasmas, Lake Arrowhead, California, USA (2015).

${ }^{26}$ M. Brix, N. C. Hawkes, A. Boboc, V. Drozdov, S. E. Sharapov, and JET-EFDA Contributors, Review of Scientific Instruments 79, (2008).

${ }^{27}$ G. T. A. Huysmans, J. P. Goedbloed, and W. Kerner, International Journal of Modern Physics C 02, 371 (1991).

${ }^{28}$ C. Hellesen, M. Gatu Johnson, E. Andersson Sundén, S. Conroy, G. Ericsson, J. Eriksson, H. Sjöstrand, M. Weiszflog, T. Johnson, G. Gorini, M. Nocente, M. Tardocchi, V. G. Kiptily, S. D. Pinches, S. E. Sharapov, and JET EFDA Contributors, Nuclear Fusion 53, 113009 (2013).

${ }^{29}$ T. H. Stix, Nuclear Fusion 15, 737 (1975).

${ }^{30}$ M. Gatu Johnson, L. Giacomelli, A. Hjalmarsson, J. Källne, M. Weiszflog, E. Andersson Sundén, S. Conroy, G. Ericsson, C. Hellesen, E. Ronchi, H. Sjöstrand, G. Gorini, M. Tardocchi, A. Combo, N. Cruz, J. Sousa, and S. Popovichev, Nuclear Instruments and Methods in Physics Research Section A: Accelerators, Spectrometers, Detectors and Associated Equipment 591, 417 (2008).

${ }^{31}$ C. Hellesen, M. Gatu Johnson, E. Anderson Sundén, S. Conroy, G. Ericsson, E. Ronchi, H. Sjöstrand, M. Weiszflog, G. Gorini, M. Tardocchi, T. Johnson, V. G. Kiptily, S. D. Pinches, S. E. Sharapov, and JET-EFDA Contributors, Nuclear Fusion 50, 022001 (2010).

${ }^{32}$ C. Hellesen, M. Gatu Johnson, E. Andersson Sundén, S. Conroy, G. Ericsson, J. Eriksson, G. Gorini, T. Johnson, V. G. Kiptily, S. D. Pinches, S. E. Sharapov, H. Sjöstrand, M. Nocente, M. Tardocchi, M. Weiszflog, and JET EFDA contributors, Nuclear Fusion 50, 084006 (2010).

${ }^{33}$ J. Eriksson, C. Hellesen, E. Andersson Sundén, M. Cecconello, S. Conroy, G. Ericsson, M. Gatu Johnson, S. D. Pinches, S. E. Sharapov, M. Weiszflog, and JET EFDA contributors, Plasma Physics and Controlled Fusion 55, 015008 (2013). 
${ }^{34}$ J. M. Adams, O. N. Jarvis, G. J. Sadler, D. B. Syme, and N. Watkins, Nuclear Instruments and Methods in Physics Research Section A: Accelerators, Spectrometers, Detectors and Associated Equipment 329, 277 (1993).

${ }^{35}$ M. Saigusa, H. Kimura, Y. Kusama, G. J. Kramer, T. Ozeki, S. Moriyama, T. Oikawa, Y. Neyatani, and T. Kondoh, Plasma Physics and Controlled Fusion 40, 1647 (1998).

${ }^{36}$ G. J. Kramer, S. E. Sharapov, R. Nazikian, N. N. Gorelenkov, and R. V. Budny, Phys. Rev. Lett. 92, 015001 (2004).

${ }^{37}$ S. Bernabei, M. G. Bell, R. V. Budny, E. D. Fredrickson, N. N. Gorelenkov, J. C. Hosea, R. Majeski, E. Mazzucato, C. K. Phillips, G. Schilling, and J. R. Wilson, Phys. Rev. Lett. 84,
$1212(2000)$

${ }^{38}$ S. Bernabei, R. Budny, E. Fredrickson, N. Gorelenkov, J. Hosea, C. Phillips, R. White, J. Wilson, C. Petty, R. Pinsker, R. Harvey, and A. Smirnov, Nuclear Fusion 41, 513 (2001).

${ }^{39}$ H. ITER Physics Expert Group on Energetic Particles, C. Drive, and I. P. B. Editors, Nuclear Fusion 39, 2471 (1999).

${ }^{40}$ Naval Research Laboratory, Plasma Formulary (2011).

${ }^{41}$ Y. Sarazin, M. Bécoulet, P. Beyer, X. Garbet, P. Ghendrih, T. C. Hender, E. Joffrin, X. Litaudon, P. J. Lomas, G. F. Matthews, V. Parail, G. Saibene, and R. Sartori, Plasma Physics and Controlled Fusion 44, 2445 (2002). 